\title{
Validation of MCNPX-PoliMi code for simulations of radioxenon beta-gamma coincidence detection
}

\author{
Ciara B. Sivels ${ }^{a, *}$, Shaun D. Clarke ${ }^{a}$, Enrico Padovani ${ }^{b}$, Amanda M. Prinke ${ }^{c}$, Justin I. McIntyre ${ }^{c}$, \\ Sara A. Pozzi ${ }^{\text {a }}$ \\ ${ }^{a}$ University of Michigan, Department of Nuclear Engineering and Radiological Sciences, Ann Arbor, MI, USA \\ ${ }^{\mathrm{b}}$ Politecnico di Milano, Department of Energy, Milan, Italy \\ c Pacific Northwest National Laboratory, Richland, WA, USA
}

\section{A R T I C L E I N F O}

\section{Keywords:}

MCNPX-PoliMi

Beta-gamma

Radioxenon

Coincidence simulation

\begin{abstract}
A B S T R A C T
Radioxenon detection is an important component of the verification regime for the Comprehensive NuclearTest-Ban Treaty. We developed and validated a new model in MCNPX-PoliMi to simulate the decay of various radioxenon isotopes and the detector response of a variety of detector types. The model was validated using calibration data from a plastic and NaI(Tl) beta-gamma coincidence detector and the results are presented. The results of this validation show that this model can also be used as a tool to produce training spectra and as a method to calibrate radioxenon detection systems.
\end{abstract}

\section{Introduction}

The Comprehensive Nuclear-Test-Ban Treaty Organization established the International Monitoring System (IMS) which consists of four technologies: infrasound, hydroacoustic, radionuclide and seismic monitoring. Radioxenon monitoring is a major component of the verification regime due to its ability to characterize an event as containing fissile material. Over the years, many detectors have been developed and tested to improve radioxenon detection capabilities [1]. A common technique for measuring radioxenon is beta-gamma coincidence detection. The detectors currently deployed in the IMS typically use a plastic scintillating cell as the beta detector and sample container, and a well-type NaI(Tl) detector as the gamma detector [2-4]. These systems have proven to be capable of measuring radioxenon from a variety of sources [5-8]. However, these systems also have limitations requiring the development of alternative detection methods. The use of coincidence detection requires advanced calibration techniques typically involving the measurement of each of the radioxenon isotopes of interest. From this calibration, the resolution of the detectors are measured and regions of interest (ROIs) are established. The ROIs are used to quantify detection efficiencies, interference ratios, and convert the beta-gamma spectra into radioxenon activities.

The goal when measuring radioxenon is to use the isotopic ratios of the sample to characterize the process generating the material. However, when using this method, civil processes sometimes overlap nuclear explosion signatures requiring better understanding of the sources of radioxenon [9]. This requires an inventory of isotopic ratios from field measurements and simulations. Detector response simulations are useful because they can provide detector information such as detection efficiency, and show the interference of one isotope with another [10], without the production of pure radioxenon samples which requires a variety of processes [11-13]. As discussed in [14], the ability to produce beta-gamma spectra can also be used for training exercises. BGSim is a program developed to produce coincidence spectra, however, the detector that is simulated cannot be changed. Therefore, alternative detector materials such as silicon and CZT, require the use of another tool to produce the coincidence spectra. Previously developed alternative tools using Geant4 or MCNP are discussed in [15-17]. The MCNP method in [15] was limited by the inability of the code to correlate the emissions of the various isotopes, requiring individual simulations for each major decay. The Geant4 methods discussed in $[16,17]$ show good agreement between simulation and experiment but require knowledge of the source code [16] and understanding of the Geant4 libraries.

MCNPX-PoliMi was developed to simulate correlated particle emissions [18]. It produces a collision file listing information about each particle history such as the detector of interaction, energy deposited, and particle type, allowing for flexible tally options. The events written to the output file are determined by specifying the detectors of interest and the minimum number of detectors in which energy is deposited. This allows for singles or only coincidence events to be collected and results in quick identification of coincidence events versus using a coincidence timing window as discussed in $[16,17]$. Since MCNP does not simulate optical photons, spectrum broadening is also included in the post-processing used to construct the histograms. This flexible

\footnotetext{
* Corresponding author.

E-mail address: csivels@umich.edu (C.B. Sivels).
} 


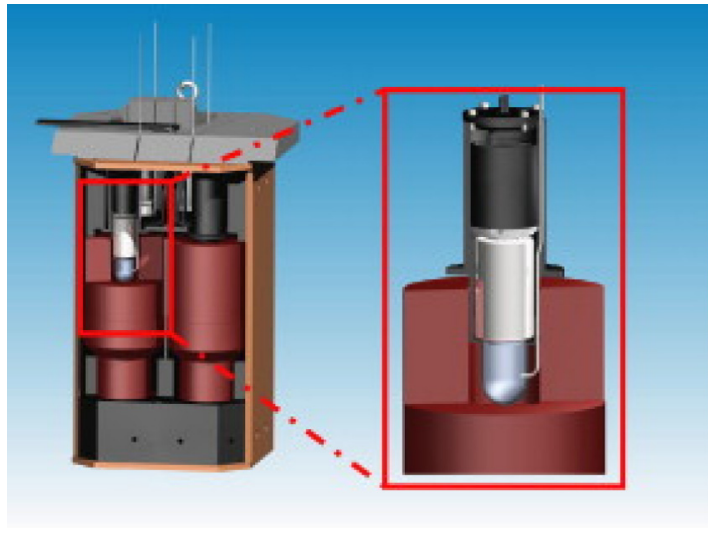

Fig. 1. NaI(Tl) well detector and rounded plastic scintillator cell [19].

approach permits a more direct generation of 2-D histograms, compared to Geant4 or MCNP6. A new built-in source, the Single Decay Option, was developed to specifically simulate radioxenon decay which the code was not able to do previously. The use of MCNPX-PoliMi, in conjunction with user-defined algorithms for detector response simulation, allows for the simulation of any detector type and geometry.

\section{Experimental setup}

There are four radioxenon isotopes of interest used to verify nuclear explosions of which two are metastable: ${ }^{135} \mathrm{Xe},{ }^{133} \mathrm{Xe},{ }^{133 \mathrm{~m}} \mathrm{Xe},{ }^{131 \mathrm{~m}} \mathrm{Xe}$. The non-metastable isotopes decay through beta emission, typically in coincidence with a gamma ray, resulting in a range of electron energies. The metastable isotopes decay through internal conversion, resulting in a specific electron energy in coincidence with typically an X-ray. For this analysis, results from a previous measurement of calibration sources were used, where the ${ }^{133 \mathrm{~m}} \mathrm{Xe}$ measurement has some ${ }^{133} \mathrm{Xe}$ mixed in due to the relationship of the isotopes. The detectors used for this study are assumed to be a rounded plastic beta cell and a well-type $\mathrm{NaI}(\mathrm{Tl})$ detector as shown in Fig. 1 [19]. The activity of each sample was estimated from these measurements using traditional methods and the simulation was based on the activity estimation. From the measurement data, the energy calibration and resolution curve were determined and used for validation.

\section{MCNPX-PoliMi simulation}

MCNPX-PoliMi has been used extensively in applications of nuclear fission $[20,21]$, but never for simulating radioxenon detection. Recent work updated the code to implement the transport of conversion electrons [22], which is vital to simulating radioxenon detection because the plastic cell is used to detect these electrons. The NaI(Tl) crystal and aluminum casing, plastic beta cell, and respective PMTs are modeled for this validation, which is similar to the model described in [22]. This analysis uses the Gaussian Energy Broadening (GEB) function to simulate the spectrum shape as used in traditional MCNP instead of the function used in [22]. The equation is a function of the full width at half maximum (FWHM) of the detector to be broadened,

$$
F W H M=a+b \sqrt{E+c E^{2}}
$$

where $a, b$, and $c$ are in units of $\mathrm{MeV}, \mathrm{MeV}$, and $1 / \mathrm{MeV}$ and $E$ is the energy of the photopeak. These coefficients are solved for by fitting the energy resolution points to Eq. (1). Therefore, a detailed calibration is required in order to obtain the best agreement between simulation and experiment. Broadening of the MCNPX-PoliMi output was done in MATLAB by randomly sampling a Gaussian distribution based on Eq. (1). The collision file output by MCNPX-PoliMi was used to tally
Table 1

Radioxenon isotopes of interest coincidence energies [24].

\begin{tabular}{lll}
\hline Isotope & Electron energy $(\mathrm{MeV})$ & Photon energy $(\mathrm{MeV})$ \\
\hline${ }^{135} \mathrm{Xe}$ & 0.915 (endpoint) & 0.250 \\
${ }^{133} \mathrm{Xe}$ & 0.346 (endpoint) & $0.030-0.035$ and 0.081 \\
${ }^{131 \mathrm{~m}} \mathrm{Xe}$ & 0.129 & $0.029-0.034$ \\
${ }^{133 \mathrm{~m}} \mathrm{Xe}$ & 0.199 & $0.029-0.034$ \\
\hline
\end{tabular}

individual and coincidence detections using MATLAB. This approach allows for spectrum generation of the individual detectors as well as their coincidence spectrum, allowing for test 2-D spectrum generation.

\section{Statistical and systematic sources of error}

The pulse height spectrum measured during an experiment is due to the randomness of radioactive decay and the properties of the detector system such as the material composition, detection mechanism, and efficiency. These properties determine the counts recorded by the detector. In radioxenon detection applications, we analyze the pulse height spectrum. The pulse height spectrum produced by the simulation is affected by statistical and systematic sources of error. The statistical error for each pulse height bin is calculated as the square root of the recorded counts,

$\sigma=\sqrt{n}$

where $n$ is the number of counts.

Sources of systematic error include the dimensions of the plastic detector and the source activity. Various plastic scintillator thicknesses were tested to analyze the effect on the pulse height spectrum, and showed that this variation has minimal effect where the beta detection efficiency remained between $95 \%-97 \%$. The true activity of the sample was unknown, which is typical for these types of experiments. The efficiency is calculated from the measured singles and coincidence ROIs and used to calculate an estimated source activity. This approach results in a source of systematic error, where the error in the singles and coincidence ROI counts varies from $\sim 2 \%-7 \%$ depending on the isotope. To estimate the calibration sample activity, previously developed calibration tools were used. Therefore, the activity uncertainty was combined with the statistical uncertainty to quantify the simulation error and is calculated using error propagation. Additionally, the $\mathrm{NaI}(\mathrm{Tl})$ reflector was not modeled, which also affects the gamma efficiency of the simulation [23].

\section{Code validation}

In this section, the simulation results are compared to experimental data for each of the isotopes of interest. The individual detector spectra are histograms of the energy deposited in either the $\mathrm{NaI}(\mathrm{Tl})$ or plastic scintillator, not accounting for interactions in the other detector. The background and gas background are subtracted for the experimental data, thus no detector background is included in the simulation. The coincidence spectra are a result of energy deposition in both the $\mathrm{NaI}(\mathrm{Tl})$ and plastic scintillator within a specified time window for the experiment or within a history for the simulation. The coincidence histogram bins energy deposited in the plastic scintillator detector on the $x$-axis and energy deposited in the $\mathrm{NaI}(\mathrm{Tl})$ detector on the $y$-axis. This approach results in a 2-D histogram where the color bar illustrates the number of counts in each bin. The most probable coincidence photon energies for each isotope are given in Table 1.

\subsection{Xenon-135}

The simulation of ${ }^{135} \mathrm{Xe}$ decay is the simplest of the four isotopes due to the emission of betas in coincidence with primarily a 250 $\mathrm{keV}$ photon. The use of Eq. (1) has improved agreement between the measurement and simulation in comparison to [22]. The NaI(Tl) 

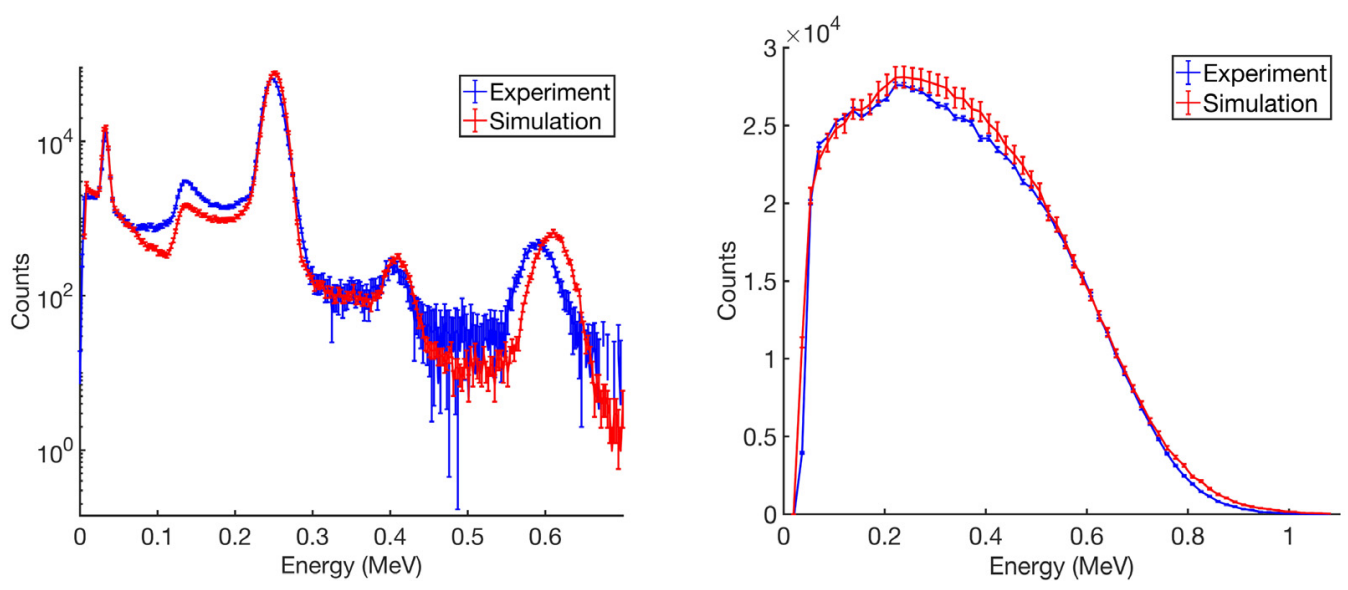

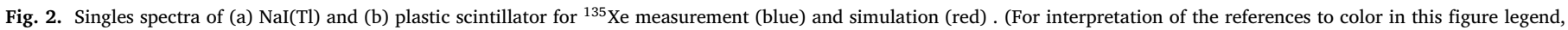
the reader is referred to the web version of this article.)
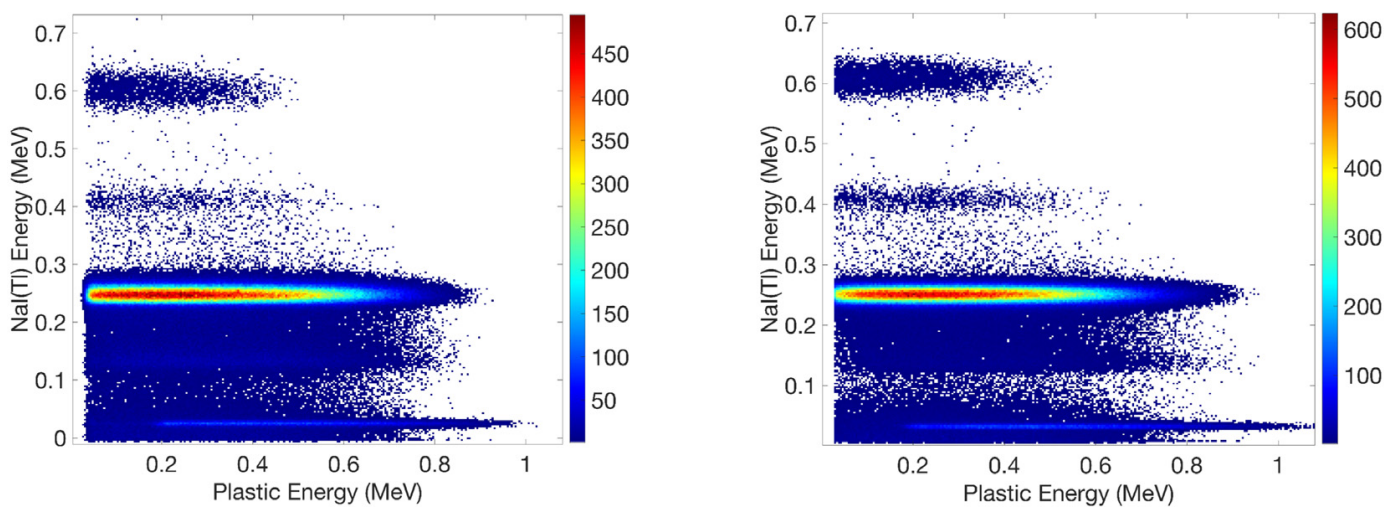

Fig. 3. Xenon-135 2-D coincidence spectra for (a) experiment and (b) simulation.
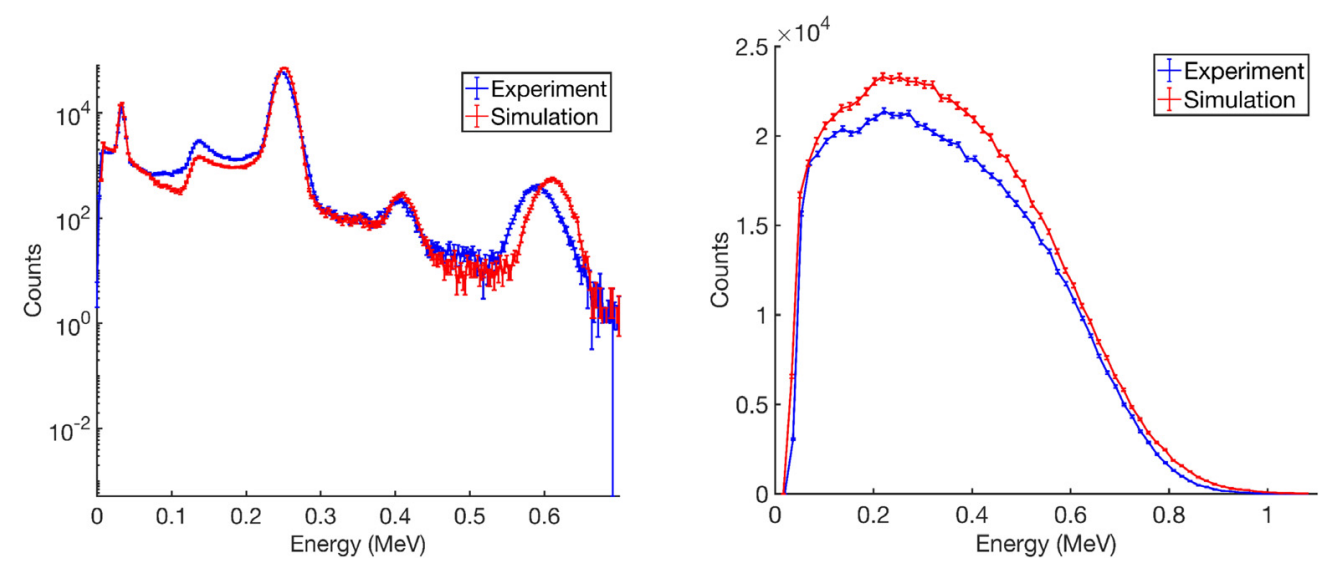

Fig. 4. Individual coincidence spectra of (a) $\mathrm{NaI}(\mathrm{Tl})$ and (b) plastic scintillator detectors for ${ }^{135} \mathrm{Xe}$.

spectrum comparison, Fig. 2a, shows good agreement between the experiment and simulation, producing two major photopeaks at 30 and $250 \mathrm{keV}$. The plastic scintillator spectrum comparison, Fig. 2b, shows the beta spectrum of ${ }^{135} \mathrm{Xe}$ which has an endpoint energy of $915 \mathrm{keV}$. The experiment and simulation spectra have similar shape with the simulation having slightly more counts, due to the activity calculation. The tail end of the beta spectrum has more counts for the simulation than the experiments and is likely due to high energy electrons escaping the plastic cell experimentally and the method in which the simulation produces a beta spectrum. The tail end of the spectrum can further be modified in MCNPX-PoliMi, but a more accurate activity calculation and simulation geometry would improve the comparison. The coincidence histograms in Fig. 3 shows similar features for the experiment and simulation with the $250 \mathrm{keV}$ ROI having the most counts and the simulation having more total coincidence counts than the experiment. Fig. 4 shows the projections of the coincidence histograms on the $x$-axis and $y$-axis for the plastic scintillator and $\mathrm{NaI}(\mathrm{Tl})$ detectors, respectively. The difference in gamma efficiency is illustrated in Fig. $4 \mathrm{~b}$ where the coincidence beta spectrum of the simulation has more counts than the experiment due to the activity estimation and the omission of the NaI(Tl) reflector. This trend is also seen for the following results. 

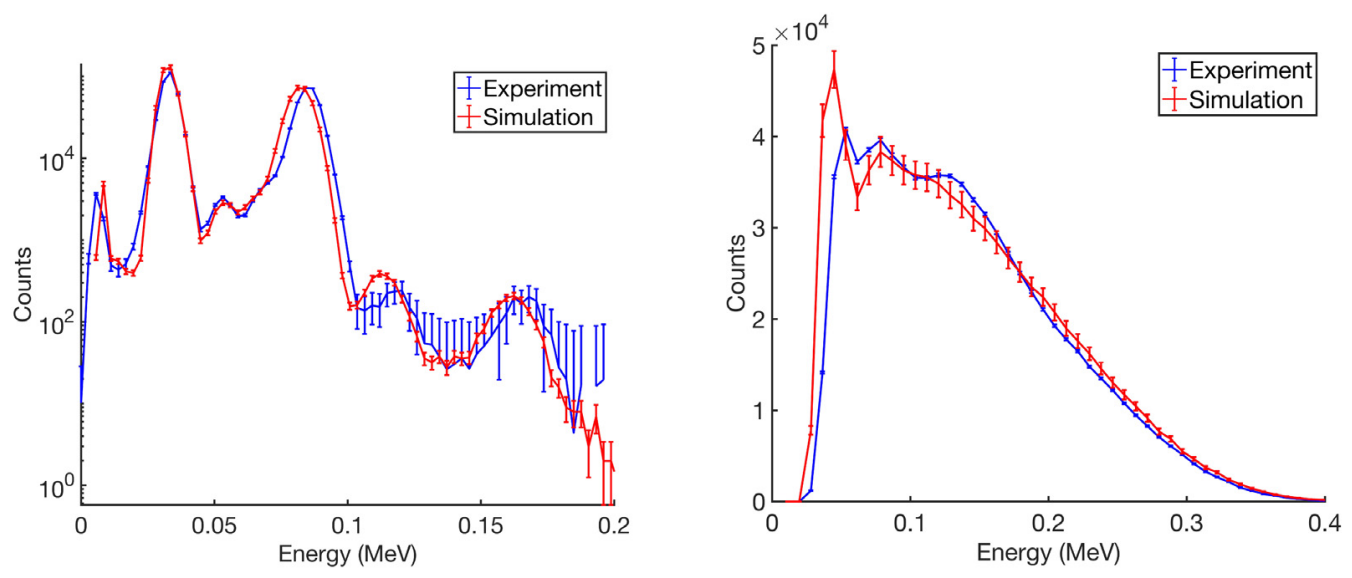

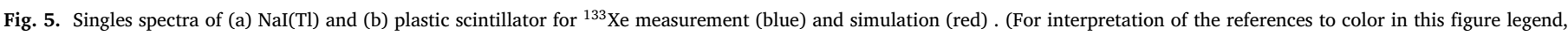
the reader is referred to the web version of this article.)
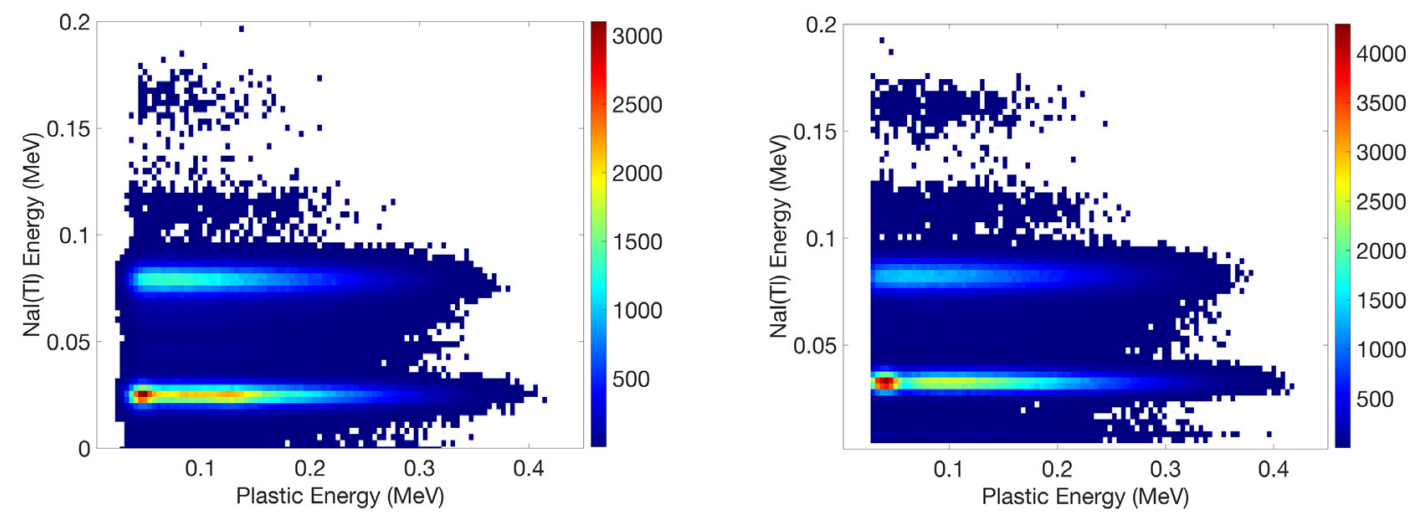

Fig. 6. Xenon-133 2-D coincidence spectra for (a) experiment and (b) simulation.
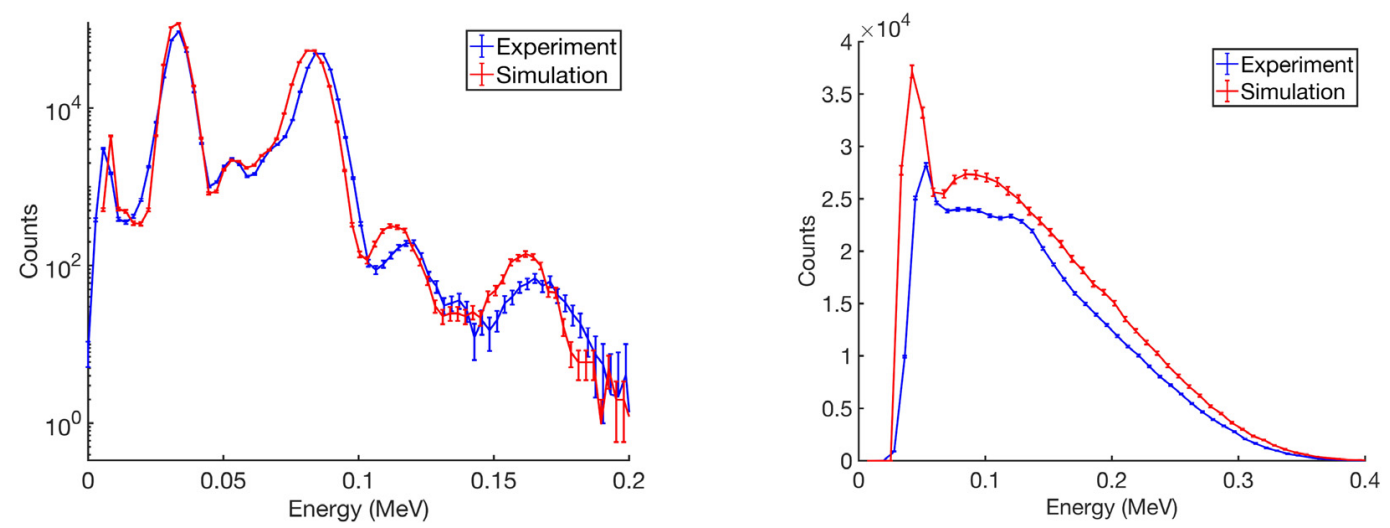

Fig. 7. Individual coincidence spectra of (a) $\mathrm{NaI}(\mathrm{Tl})$ and (b) plastic scintillator detectors for ${ }^{133} \mathrm{Xe}$.

\subsection{Xenon-133}

The simulation of ${ }^{133} \mathrm{Xe}$ is more challenging than ${ }^{135} \mathrm{Xe}$ due to its complex decay scheme, which often includes a beta emission followed by a coincidence gamma-ray or coincidence conversion electron and $\mathrm{X}$-ray. Additionally, the sample is produced through fission, which results in a small amount of ${ }^{131 \mathrm{~m} X e}$ contamination that was not added to the simulation. The $\mathrm{NaI}(\mathrm{Tl})$ spectrum comparison, Fig. 5a, shows good agreement between the experiment and simulation, producing two major photopeaks at 30 and $81 \mathrm{keV}$. Fig. 5b shows the beta spectra comparison where the $45 \mathrm{keV}$ conversion electron peak is much higher for the simulation than experiment due to threshold limitations of the simulation. Otherwise, the beta spectrum shape is similar between the experiment and simulation. Fig. 6 shows the coincidence histogram of the experiment and simulation, with high count regions for the beta coincidences with 30 and $81 \mathrm{keV}$ photons, with a slight bump in the experimental coincidence histogram at $\sim 130 \mathrm{keV}$ for the plastic (x-axis) and $30 \mathrm{keV}$ for the $\mathrm{NaI}(\mathrm{Tl})$ ( $y$-axis) due to the ${ }^{131 \mathrm{~m}} \mathrm{Xe}$ contamination. The projected coincidence spectra are shown in Fig. 7. The NaI(Tl) spectra in Fig. 7a have similar features with the simulation again having more counts. The beta coincidence spectrum of the simulation also has more counts again due to the higher gamma efficiency of the simulation compared to the experiment. The contamination of ${ }^{131 \mathrm{~m} X e}$ is also producing a slight bump at $129 \mathrm{keV}$ for the experiment in Fig. $7 \mathrm{~b}$. 

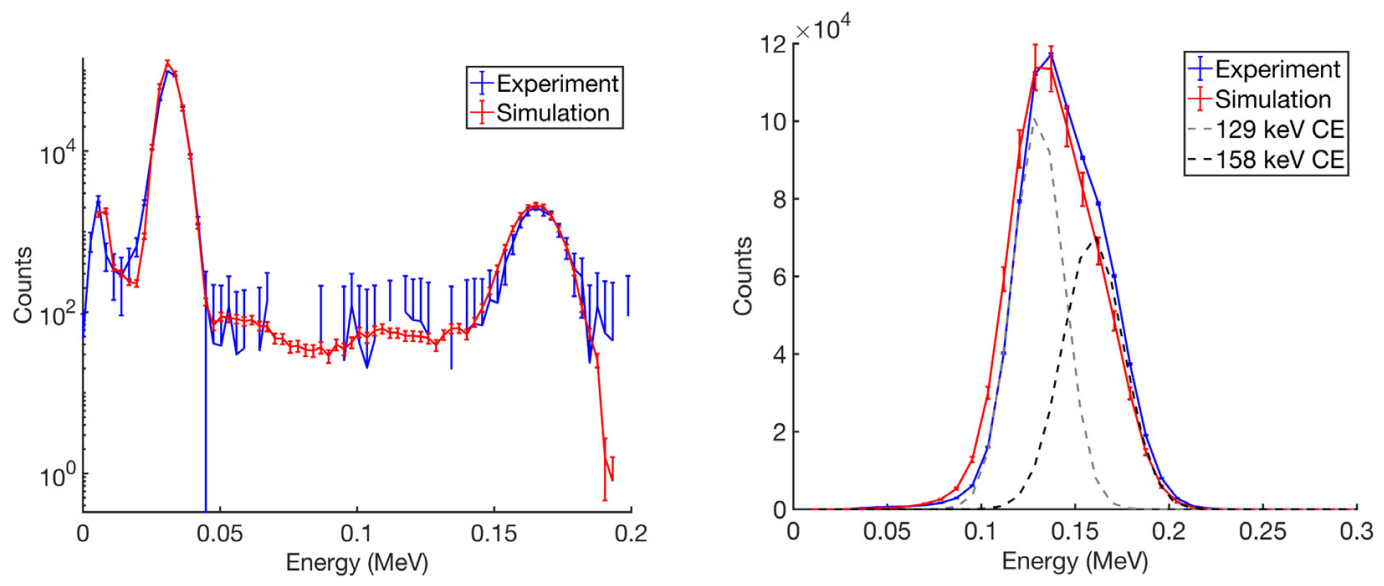

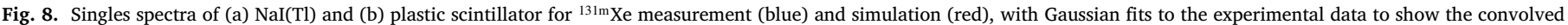
$129 \mathrm{keV}$ and $158 \mathrm{keV}$ conversion electron energy peaks. (For interpretation of the references to color in this figure legend, the reader is referred to the web version of this article.)
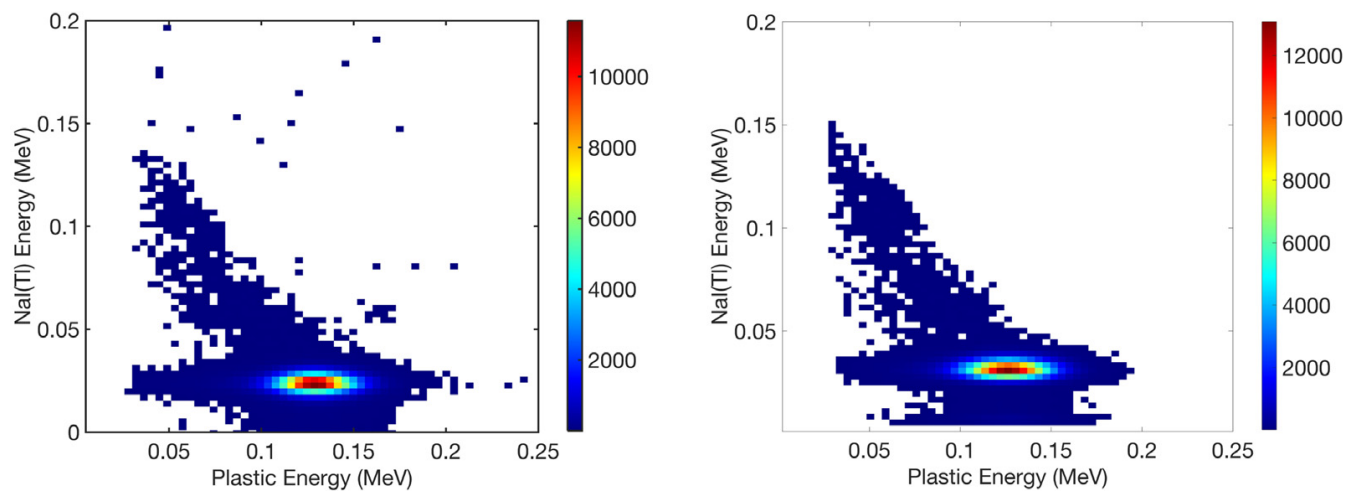

Fig. 9. Xenon-131m 2-D coincidence spectra for the (a) experiment and (b) simulation.
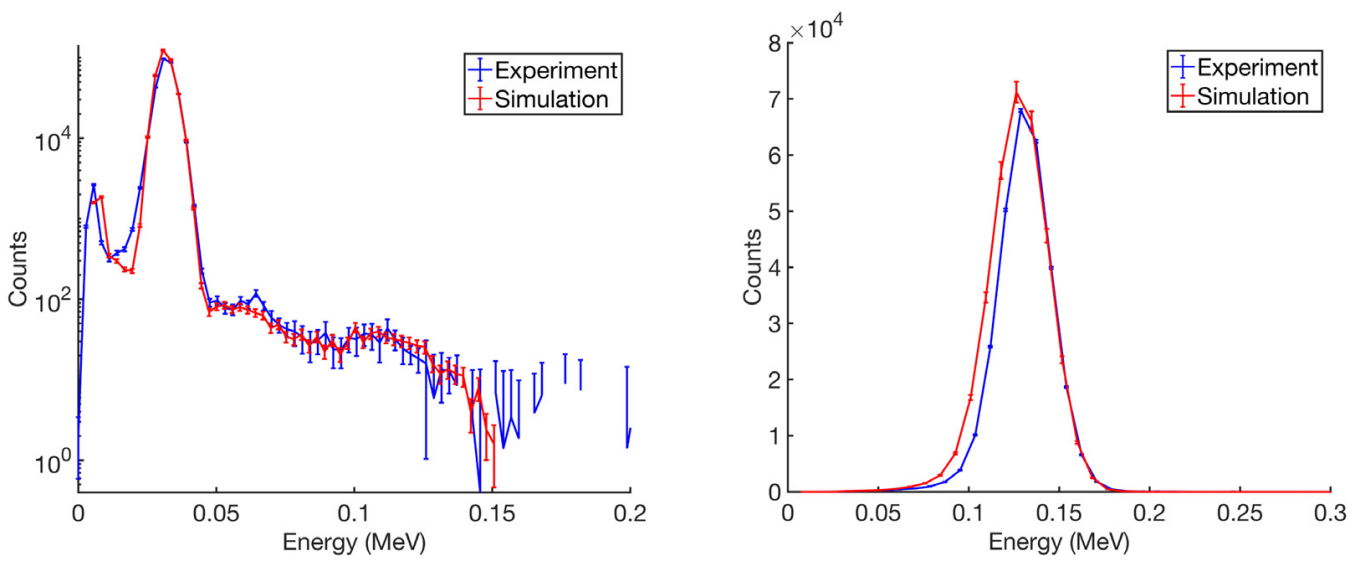

Fig. 10. Individual coincidence spectra of the (a) $\mathrm{NaI}(\mathrm{Tl})$ and (b) plastic scintillator detectors for ${ }^{131 \mathrm{~m}} \mathrm{Xe}$.

\subsection{Xenon- $131 m$}

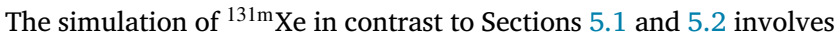
distinct $\mathrm{CE}$ energies. The NaI(Tl) spectrum comparison, Fig. 8a, shows similar features for the experiment and simulation, producing $30 \mathrm{keV}$ and $163 \mathrm{keV}$ photopeaks. The plastic spectrum comparison, Fig. 8b, shows experimental and simulation agreement and Gaussian fits of the 129 and 158 CE peaks for the experiment. Fig. 9 shows almost identical features with the highest counts in the $129 \mathrm{keV}$ ROI and the simulation having more counts, minor differences in the size of the circle are due to energy resolution broadening. The coincidence spectra projections are shown in Fig. 10. Again, the gamma efficiency for the simulation is higher resulting in more counts in the coincidence beta spectrum as shown in Fig. 10b. However, agreement has greatly improved with the use of Eq. (1) and updates to the source compared to results shown in [22].

\subsection{Mixed xenon-133m, xenon-133, and xenon-135}

The decay of ${ }^{133 m} \mathrm{Xe}$ is more complex to simulate because it is constantly decaying into ${ }^{133} \mathrm{Xe}$. Additionally, there was some ${ }^{135} \mathrm{Xe}$ still present in the cell that had to be accounted for. This simulation 

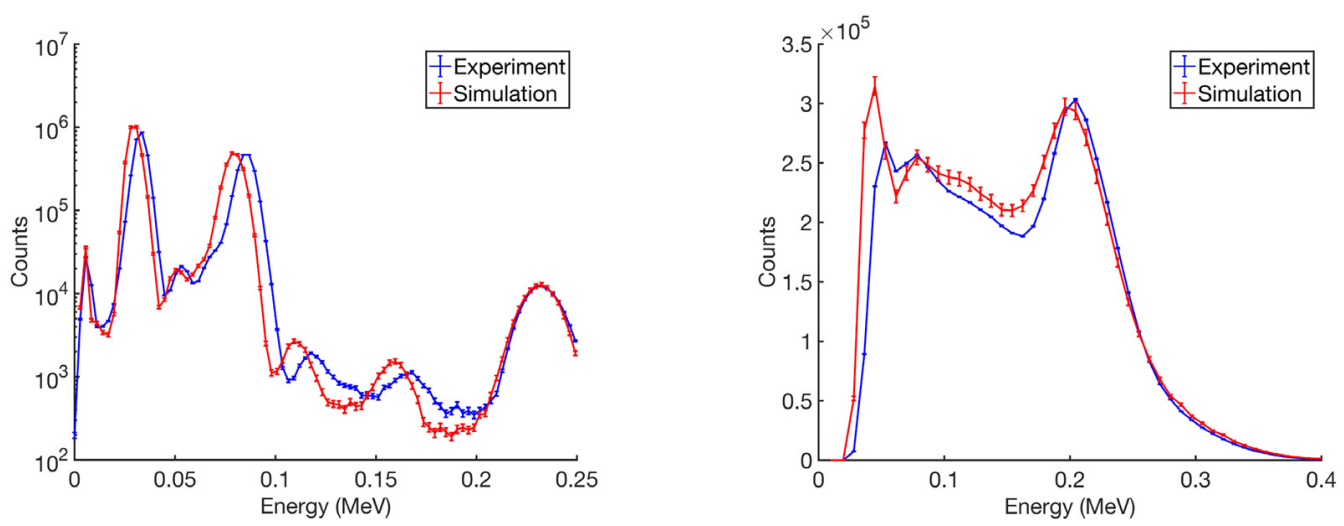

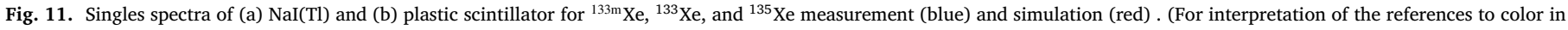
this figure legend, the reader is referred to the web version of this article.)
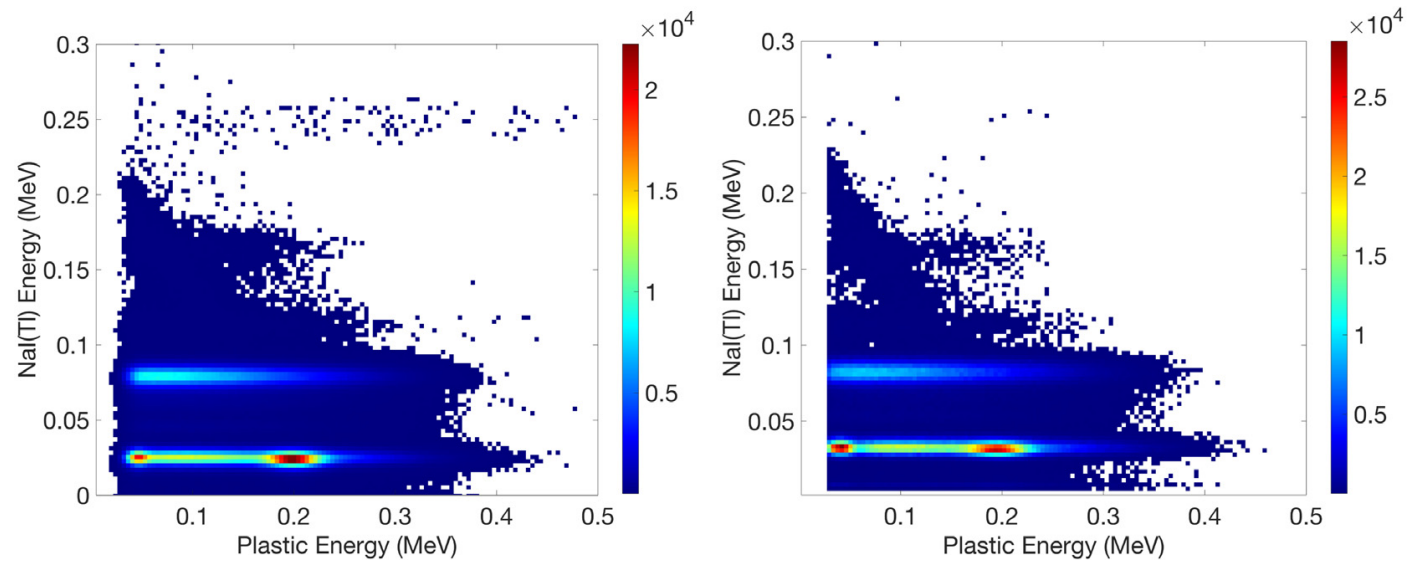

Fig. 12. Xenon-133m, xenon-133, and xenon-135 2-D coincidence spectra for (a) experiment and (b) simulation.
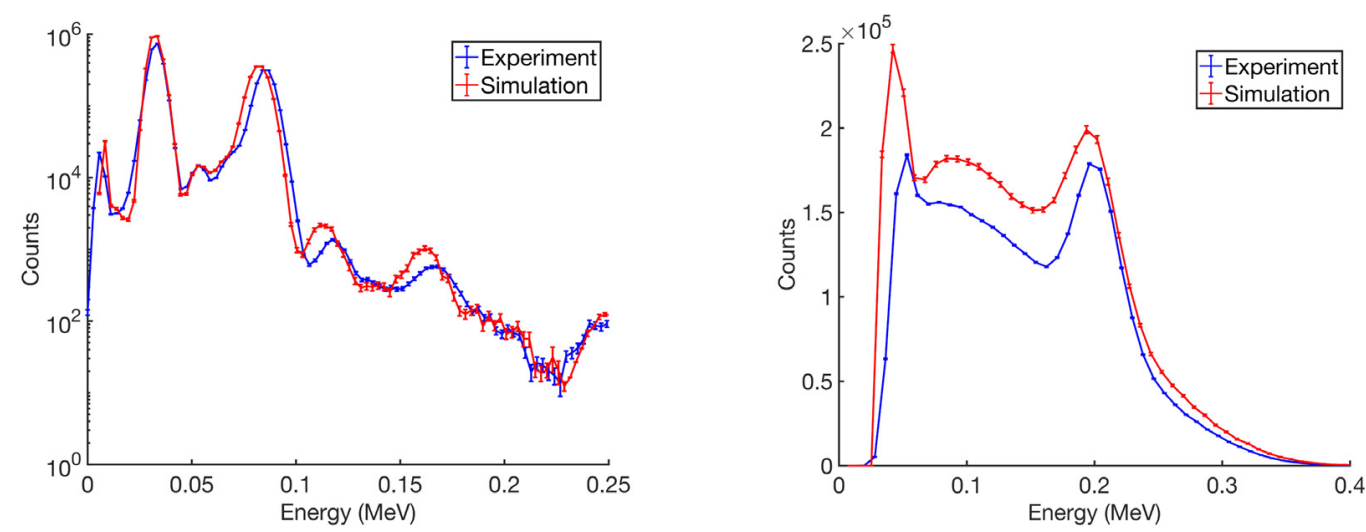

Fig. 13. Individual coincidence spectra of the $\mathrm{NaI}(\mathrm{Tl})$ (a) and plastic scintillator (b) detectors for mixed ${ }^{133 \mathrm{~m}} \mathrm{Xe},{ }^{133} \mathrm{Xe}$, and ${ }^{135} \mathrm{Xe}$.

required the combination of individual simulations of ${ }^{135} \mathrm{Xe},{ }^{133} \mathrm{Xe}$, and ${ }^{133 m}$ Xe using estimated activities. In contrast to the ${ }^{133} \mathrm{Xe}$ gamma spectra shown in Fig. 5, the ${ }^{133 \mathrm{~m}} \mathrm{Xe}$ isotope also produces a $233 \mathrm{keV}$ photon as shown on in Fig. 11a. From ${ }^{133} \mathrm{Xe}$ decay, the $30 \mathrm{keV}$ and $81 \mathrm{keV}$ peaks are also present in the plot. The beta spectrum comparison, shown in Fig. 11b, again overestimates the $45 \mathrm{keV}$ conversion electron. However, there is good agreement between the experiment and simulation for the $199 \mathrm{keV}$ conversion electron and the endpoint energies align fairly well. The coincidence spectrum, Fig. 12, shows the ROIs due to ${ }^{133} \mathrm{Xe}$ as well as the $199 \mathrm{keV}$ ROI from ${ }^{133 \mathrm{~m}} \mathrm{Xe}$, illustrating the ability of this tool to simulate the detector response of mixed samples. There is an additional feature at the $250 \mathrm{keV}$ gamma line due to the ${ }^{135} \mathrm{Xe}$ presence in the experiment. Projections of the coincidence histogram are shown in Fig. 13. The NaI(Tl) spectra in Fig. 13a show similar features to that of Fig. 11a with the simulation having more counts. The beta spectrum in Fig. 13b have similar features but again show that the higher gamma efficiency of the simulation results in more counts in the coincidence spectrum of the simulation.

\section{Conclusions}

We demonstrate and validate the use of a new Single Decay Option in MCNPX-PoliMi to simulate radioxenon decay using plastic and $\mathrm{NaI}(\mathrm{Tl})$ scintillators in a coincidence set-up. The results presented here include 
simulations of ${ }^{135} \mathrm{Xe},{ }^{133} \mathrm{Xe},{ }^{131 \mathrm{~m}} \mathrm{Xe}$, and a mixed source of ${ }^{133 \mathrm{~m}} \mathrm{Xe}$, ${ }^{133} \mathrm{Xe}$, and ${ }^{135} \mathrm{Xe}$ and serve as validation of this tool for radioxenon detection in Comprehensive Nuclear-Test-Ban Treaty verification applications. The results show that the new tool is able to simulate the shape and magnitude of radioxenon signatures for both singles and coincidence mode. The observed differences between experiment and simulation were a result of having to estimate the activity of the sample and simplifications of the model. We also found that limitations arise if the resolution of the detector is not well known.

Further developments include the implementation of modified beta spectrum tails, low-energy X-ray emissions, light transport effects, and careful accounting of variances in detector geometry. This tool could be used to simplify current calibration measurements through simulation of the detector response, requiring fewer radioxenon measurements. Additionally, as shown with the mixed sample, this tool can be used to produce coincidence spectra for a variety of detector types with the purpose of training radionuclide analysts, as well as assisting in the analysis of measured samples such as those taken during On-Site Inspection training.

\section{Acknowledgments}

This work was funded in-part by the Consortium for Verification Technology under Department of Energy National Nuclear Security Administration award number DE-NA0002534. We would also like to thank the staff at PNNL for providing experimental data and technical support.

\section{References}

[1] C.B. Sivels, J.I. McIntyre, T.W. Bowyer, M.B. Kalinowski, S.A. Pozzi, A review of the developments of radioxenon detectors for nuclear explosion monitoring, J. Radioanal. Nucl. Chem. 314 (2) (2017) 829-841. http://dx.doi.org/10.1007/ s10967-017-5489-2.

[2] A. Ringbom, T. Larson, A. Axelsson, K. Elmgren, C. Johansson, SAUNA-a system for automatic sampling, processing, and analysis of radioactive xenon, Nucl. Instrum. Methods Phys. Res. A 508 (3) (2003) 542-553. http://dx.doi.org/10.1016/S01689002(03)01657-7.

[3] J.I. McIntyre, K.H. Abel, T.W. Bowyer, J.C. Hayes, T.R. Heimbigner, M.E. Panisko, P.L. Reeder, R.C. Thompson, Measurements of ambient radioxenon levels using the automated radioxenon sampler/analyzer (ARSA), J. Radioanal. Nucl. Chem. 248 (3) (2001) 629-635. http://dx.doi.org/10.1023/A:1010672107749.

[4] V.V. Prelovskii, N.M. Kazarinov, A.Y. Donets, V.Y. Popov, I.Y. Popov, N.V. Skirda, The ARIX-03f mobile semiautomatic facility for measuring low concentrations of radioactive xenon isotopes in air and subsoil gas, Instrum. Exp. Tech. 50 (3) (2007) 393-397. http://dx.doi.org/10.1134/S0020441207030165.

[5] G. Wotawa, A. Becker, M. Kalinowski, P. Saey, M. Tuma, M. Zahringer, Computation and analysis of the global distribution of the radioxenon isotope 133Xe based on emissions from nuclear power plants and radioisotope production facilities and its relevance for the verification of the nuclear-test-ban treaty, Pure Appl. Geophys. 167 (4) (2010) 541-557. http://dx.doi.org/10.1007/s00024-009-0033-0.

[6] P.R. Saey, T.W. Bowyer, A. Ringbom, Isotopic noble gas signatures released from medical isotope production facilities-simulations and measurements, Appl. Radiat. Isot. 68 (9) (2010) 1846-1854. http://dx.doi.org/10.1016/j.apradiso.2010.04.014.

[7] A. Stohl, P. Seibert, G. Wotawa, The total release of xenon-133 from the fukushima dai-ichi nuclear power plant accident, J. Environ. Radioact. 112 (2012) 155-159. http://dx.doi.org/10.1016/j.jenvrad.2012.06.001.
[8] A. Ringbom, A. Axelsson, M. Aldener, M. Auer, T. Bowyer, T. Fritioff, I. Hoffman, K. Khrustalev, M. Nikkinen, V. Popov, Y. Popov, K. Ungar, G. Wotawa, Radioxenon detections in the CTBT international monitoring system likely related to the announced nuclear test in North Korea on February 12, 2013, J. Environ. Radioact. 128 (2014) 47-63. http://dx.doi.org/10.1016/j.jenvrad.2013.10.027.

[9] M.B. Kalinowski, A. Axelsson, M. Bean, X. Blanchard, T.W. Bowyer, G. Brachet, S. Hebel, J.I. McIntyre, J. Peters, C. Pistner, M. Raith, A. Ringbom, P.R.J. Saey, C. Schlosser, T.J. Stocki, T. Taffary, R. Kurt Ungar, Discrimination of nuclear explosions against civilian sources based on atmospheric xenon isotopic activity ratios, Pure Appl. Geophys. 167 (4) (2010) 517-539. http://dx.doi.org/10.1007/ s00024-009-0032-1.

[10] J.H. Ely, M.W. Cooper, D.A. Haas, J.C. Hayes, T.R. Heimbigner, B.T. Schrom, Interference terms for xenon-135, in: Proceedings of the 2011 Monitoring Research Review: Ground-Based Nuclear Explosion Monitoring Technologies, 1, Pacific Northwest National Laboratory, Richland, WA, United States (US), Los Alamos National Laboratory, Los Alamos, NM, United States (US), 2011, pp. 664-670.

[11] M.G. Watrous, J.E. Delmore, R.K. Hague, T.P. Houghton, D.D. Jenson, N.R. Mann, Radioxenon spiked air, J. Environ. Radioact. 150 (2015) 126-131. http://dx.doi. org/10.1016/j.jenvrad.2015.08.005.

[12] H. Gohla, M. Auer, P. Cassette, R. Hague, M. Lechermann, B. Nadalut, Radioxenon standards used in laboratory inter-comparisons, in: Proceedings of the 20th International Conference on Radionuclide Metrology and its Applications 8-11 2015, Vienna, Austria, Appl. Radiat. Isot. 109 (2016) 24-29. http://dx.doi.org/10.1016/ j.apradiso.2015.11.044.

[13] S.R. Biegalski, T.N. Tipping, F.J. Klingberg, Preparation of radioxenon and radioargon mixed sources for ife14, J. Radioanal. Nucl. Chem. 307 (3) (2016) 1837-1840. http://dx.doi.org/10.1007/s10967-015-4398-5.

[14] J.I. McIntyre, B.T. Schrom, M.W. Cooper, A.M. Prinke, T.J. Suckow, A. Ringbom, G.A. Warren, A program to generate simulated radioxenon beta-gamma data for concentration verification and validation and training exercises, J. Radioanal. Nucl. Chem. 307 (3) (2016) 2381-2387. http://dx.doi.org/10.1007/s10967-015-46205.

[15] D.A. Haas, S.R. Biegalski, K.M. Foltz Biegalski, Modeling $\beta-\gamma$ coincidence spectra of ${ }^{131 \mathrm{~m}} \mathrm{Xe},{ }^{133} \mathrm{Xe},{ }^{133 \mathrm{~m}} \mathrm{Xe}$, and ${ }^{135} \mathrm{Xe}$, J. Radioanal. Nucl. Chem. 277 (3) (2008) 561. http://dx.doi.org/10.1007/s10967-007-7178-z.

[16] W. Zhang, P. Mekarski, J. Lam, K. Ungar, A Geant4 Monte Carlo method for synthesizing radioxenon beta-gamma coincidence spectra, J. Radioanal. Nucl. Chem. 286 (1) (2010) 235-240. http://dx.doi.org/10.1007/s10967-010-0645-y.

[17] V. Doost-Mohammadi, H. Afarideh, G.R. Etaati, Simulation of beta-gamma coincidence spectra of radioxenon detector using gate 7.0 and comparison with experimental results, J. Radioanal. Nucl. Chem. 309 (3) (2016) 1105-1112. http: //dx.doi.org/10.1007/s10967-016-4720-x.

[18] S.A. Pozzi, E. Padovani, M. Marseguerra, MCNP-PoliMi: a Monte-Carlo code for correlation measurements, Nucl. Instrum. Methods Phys. Res. A 513 (3) (2003) 550-558. http://dx.doi.org/10.1016/j.nima.2003.06.012.

[19] M.W. Cooper, J.I. McIntyre, T.W. Bowyer, A.J. Carman, J.C. Hayes, T.R. Heimbigner, C.W. Hubbard, L. Lidey, K.E. Litke, S.J. Morris, M.D. Ripplinger, R. Suarez, R. Thompson, Redesigned $\beta-\gamma$ radioxenon detector, Nucl. Instrum. Methods Phys. Res. A 579 (2007) 426-430. http://dx.doi.org/10.1016/j.nima.2007.04.092.

[20] M.C. Hamel, J.K. Polack, M.C. Ruch, M.J. Marcath, S.D. Clarke, S.A. Pozzi, Active neutron and gamma-ray imaging of highly enriched uranium for treaty verification, Sci. Rep. 7 (2017) 7997. http://dx.doi.org/10.1038/s41598-017-08253-x.

[21] T.H. Shin, M.Y. Hua, M.J. Marcath, D.L. Chichester, I. Pázsit, A. Di Fulvio, S.D. Clarke, S.A. Pozzi, Neutron multiplicity counting moments for fissile mass estimation in scatter-based neutron detection systems, Nucl. Sci. Eng. 188 (3) (2017) 246-269. http://dx.doi.org/10.1080/00295639.2017.1354591.

[22] C.B. Sivels, S.D. Clarke, S.A. Pozzi, E. Padovani, MCNPX-PoliMi simulation of radioxenon detection for treaty verification, in: Institute of Nuclear Materials Management 58th Annual Meeting, Indian Wells, California, July 2017, pp. 16-20.

[23] H.D. Tam, H.D. Chuong, T.T. Thanh, C.V. Tao, A study of the effect of Al2O3 reflector on response function of $\mathrm{NaI}(\mathrm{Tl})$ detector, Radiat. Phys. Chem. 125 (2011) 88-93. http://dx.doi.org/10.1016/j.radphyschem.2016.03.020.

[24] R.B. Firestone, C.M. Baglin, S. Chu, Table of Isotopes, No. V. 4, Wiley, 1999, https://books.google.com/books?id = W4JUAAAAMAAJ. 